\title{
Relationship among Corneal Biomechanics, Anterior Segment Parameters, and Geometric Corneal Parameters
}

\author{
Sadık Görkem Çevik, ${ }^{1}$ Sertaç Argun Kıvanç, ${ }^{2}$ Berna Akova-Budak, ${ }^{2}$ and Mediha Tok-Çevik ${ }^{3}$ \\ ${ }^{1}$ Department of Ophthalmology, Bursa Yuksek Ihtisas Training and Research Hospital, Bursa, Turkey \\ ${ }^{2}$ Department of Ophthalmology, School of Medicine, Uludag University, Bursa, Turkey \\ ${ }^{3}$ Department of Ophthalmology, Düzici State Hospital, Düzici, Turkey
}

Correspondence should be addressed to Sertaç Argun Kıvanç; sakivanc@gmail.com

Received 25 June 2016; Revised 7 September 2016; Accepted 14 September 2016

Academic Editor: Kin Sheng Lim

Copyright (C) 2016 Sadık Görkem Çevik et al. This is an open access article distributed under the Creative Commons Attribution License, which permits unrestricted use, distribution, and reproduction in any medium, provided the original work is properly cited.

Purpose. To investigate the relationship between corneal biomechanical parameters, anterior segment parameters, and geometric corneal parameters in a healthy Caucasian group. Methods. This retrospective study included the healthy eyes with best corrected visual acuity of at least 20/40 of 122 Caucasian subjects. The anterior segment parameters and geometric corneal parameters such as corneal volume, central corneal thickness, horizontal and vertical corneal radii, anterior and posterior steep, and flat keratometric values were measured with a Scheimpflug camera. The biomechanical properties were measured with Ocular Response Analyzer. Results. One hundred and twenty-two healthy Caucasian subjects (67 males, 55 females) with a mean age of $45.32 \pm 20.23$ were enrolled. Both corneal hysteresis and corneal resistance factor were positively correlated with CCT $(r=0.529, p<0.001 ; r=0.638$, $p<0.001)$ and CV $(r=0.635, p<0.001 ; r=0.579, p<0.001)$ and negatively correlated with age $(r=-0.373, p<0.001$; $r=-0.249, p<0.001)$. Both in age-gender and multivariate models, $\mathrm{CH}$ and CRF had statistically significant negative association with the posterior steep $K$ value. Conclusions. $\mathrm{CH}$ and CRF are negatively correlated with posterior steep and average posterior $K$ values.

\section{Introduction}

Many factors affect corneal biomechanical properties in healthy eyes [1-3]. Anterior segment parameters such as central corneal thickness (CCT) are among these factors that have been intensively studied. Corneal hysteresis $(\mathrm{CH})$ and corneal resistance factor (CRF) are strongly correlated with CCT [4-6]. A relation between refractive error and corneal biomechanical properties has also been reported [7]. The relationship between other anterior segment parameters such as corneal curvature (CC), corneal astigmatism (CA), corneal volume $(\mathrm{CV})$, mean keratometric $(K)$ value, and corneal biomechanical properties has been also investigated and the results are controversial [8-11]. Several studies in children reported that flatter corneal curvature was related to lower $\mathrm{CH}$ and $\mathrm{CRF}$ values $[12,13]$. In this study, we aimed to investigate the relationship between corneal biomechanical parameters, anterior segment parameters, and geometric corneal parameters, including horizontal and vertical corneal radii and anterior and posterior steep and flat $K$ values with $3 \mathrm{~mm}$ distance from the apex in a healthy Caucasian group.

\section{Methods}

2.1. Subjects. This retrospective study was carried out at Department of Ophthalmology of Bursa Yukses Ihtisas Training and Research Hospital. Initially, the medical records of 158 patients with best corrected visual acuity of at least 20/40 in one eye were reviewed. These patients were referred to the department for assessment of eligibility for refractive surgery, renewal of driving license, or obtaining medical report for any reason. The investigation was conducted in accordance with the Declaration of Helsinki and approved by institution. The exclusion criteria were history of previous ocular surgery and contact lens wearing, ocular inflammation, glaucoma, use of topical medication, and presence of diabetes mellitus and collagenous diseases. Of 158 patients, 122 healthy eyes of 122 subjects were included in the study. 
TABLE 1: The biomechanical properties, anterior segment parameters, and refraction values of healthy subjects.

\begin{tabular}{lcc}
\hline & Mean & Standard deviation \\
\hline Age (year) & 45.32 & 20.23 \\
$\mathrm{CH}(\mathrm{mmHg})$ & 10.67 & 1.86 \\
$\mathrm{CRF}(\mathrm{mmHg})$ & 10.89 & 1.79 \\
$\mathrm{IOPg}(\mathrm{mmHg})$ & 16.35 & 4.08 \\
$\mathrm{IOPc}(\mathrm{mmHg})$ & 16.33 & 4.26 \\
$\mathrm{CCT}\left(\mathrm{microns}^{2}\right.$ & 552.19 & 37.21 \\
$\mathrm{CV}\left(\mathrm{mm}^{3}\right)$ & 60.69 & 4.42 \\
ACD $\left(\mathrm{mm}^{3}\right)$ & 2.79 & 0.52 \\
ACV (mm $\left.{ }^{3}\right)$ & 161.06 & 47.19 \\
Spherical value (D) & -0.34 & 2.45 \\
Cylindrical value (D) & -0.75 & 0.56 \\
SE (D) & -.54 & 2.15 \\
\hline
\end{tabular}

CH: corneal hysteresis; CRF: corneal resistance factor; IOPgc: intraocular pressure (Goldman correlated); IOPcc: intraocular pressure (cornea compensated); CCT: central corneal thickness; CV: corneal volume; ACD: anterior chamber depth; ACV: anterior chamber volume; SE: spherical equivalent, D: diopter.

2.2. Ocular Examinations. All the subjects underwent complete ophthalmologic examination including refraction and visual acuity measurement, biomicroscopic examination, intraocular pressure (IOP) measurement with a Goldmann applanation tonometer, and funduscopy.

2.3. Corneal Topography and Thickness Measurement. The anterior segment of the included eye of each subject was imaged with a 3D rotating Scheimpflug camera Sirius (CSO, Costruzione Strumenti Oftalmici, Florence, Italy) without application of any eyedrops. One of the authors performed all the measurements with Sirius. CCT, CV, anterior chamber depth (ACD), anterior chamber volume (ACV), horizontal and vertical corneal radii, $C A$, mean corneal $K$, and anterior and posterior corneal $K$ values with $3 \mathrm{~mm}$ distance from the apex were measured.

2.4. Ocular Response Analyzer (ORA). Four consecutive ORA (Reichert, Buffalo, New York, USA) measurements were performed and the averages of the measurements were taken. The high-quality readings defined by the manufacturer, because the force-in and force-out applanation signal peaks on the ORA waveform are fairly symmetrical in height, were accepted and recorded. The parameters used for analysis were $\mathrm{CH}$, CRF, corneal compensated IOP (IOPcc), and Goldmann correlated IOP (IOPgc).

2.5. Statistical Analysis. The statistical analysis was performed by SPSS 22.0 statistical program. The relationship between variables was assessed with Pearson correlation analysis. Kolmogorov-Smirnov test was performed for the assessment of normal distribution of the data. Multivariate linear regression analysis was used for assessment of association of $\mathrm{CH}, \mathrm{CRF}$ with anterior segment parameters, and geometric corneal parameters, including horizontal and vertical corneal radii, anterior and posterior steep and flat $K$ values, IOP, and spherical equivalent.

\section{Results}

One hundred and twenty-two healthy Caucasian subjects (67 males, 55 females) with a mean age of $45.32 \pm 20.23$ were included in the study. The biomechanical properties, anterior segment parameters, and refraction values are given in Table 1. Table 2 shows the relationship of $\mathrm{CH}, \mathrm{CRF}, \mathrm{CCT}$, and $\mathrm{CV}$ with IOPgc, IOPcc, anterior segment parameters, and refraction values. Both $\mathrm{CH}$ and $\mathrm{CRF}$ were positively correlated with CCT $(r=0.529, p<0.001 ; r=0.638$, $p<0.001)$ and CV $(r=0.635, p<0.001 ; r=0.579$, $p<0.001)$ and negatively correlated with age $(r=-0.373$, $p<0.001 ; r=-0.249, p<0.001)$. The corneal curvature and $K$ values of both anterior and posterior surfaces are listed in Table 3. Correlations were found between $\mathrm{CH}$ and mean anterior $K$ value, anterior flat $K$ value, mean posterior $K$ value, posterior steep $K$ value, and vertical radius of CC. CRF was also correlated with mean posterior $K$ value and posterior steep $K$ value. No correlation was detected between $\mathrm{CH}$, CRF, and mean $K$ value (Table 4 ). In multivariate linear regression analysis, the associations between the corneal biomechanical parameters with $K$ values of anterior and posterior surfaces and anterior segment parameters were investigated (Tables 5 and 6). Both in age-gender and multivariate models, $\mathrm{CH}$ and CRF had statistically significant negative association with the posterior step $K$ value (Table 5). When IOP, ACD, ACV, $\mathrm{CV}$, CCT, and SE were included in multivariate analysis, no association was detected between corneal biomechanical properties and $K$ values. Age and IOP were negatively ( $r=$ $-0.275, p=0.016 ; r=-0.174, p=0.048$, resp.) and CCT was positively associated with $\mathrm{CH}(r=0.477, p=0.043)$. In addition, there were positive associations between IOP, ACD, and CCT with CRF $(r=0.323, p<0.001 ; r=0.350$, $p=0.028 ; r=0.741, p=0.001$, resp.) (Table 6).

\section{Discussion}

Previous studies showed that there is a correlation between corneal biomechanical properties and age. Though there 
TABLE 2: The relationship of CH, CRF, CCT, and CV with IOPgc, IOPcc, anterior segment parameters, and refraction values.

\begin{tabular}{lcccccccccc}
\hline & \multicolumn{2}{c}{ Age } & \multicolumn{2}{c}{ CH } & \multicolumn{2}{c}{ CRF } & \multicolumn{2}{c}{ CCT } & \multicolumn{2}{c}{ CV } \\
& $r$ value & $p$ value & $r$ value & $p$ value & $r$ value & $p$ value & $r$ value & $p$ value & $r$ value & $p$ value \\
\hline CH & -0.373 & $<0.001$ & N/A & N/A & 0.752 & $<0.001$ & 0.529 & $<0.001$ & 0.635 & $<0.001$ \\
CRF & -0.249 & 0.015 & 0.752 & $<0.001$ & N/A & N/A & 0.638 & $<0.001$ & 0.579 & $<0.001$ \\
IOPgc & 0.137 & 0.185 & -0.202 & 0.046 & 0.506 & $<0.001$ & 0.247 & 0.014 & 0.041 & 0.692 \\
IOPcc & 0.279 & 0.005 & -0.620 & $<0.001$ & 0.066 & 0.517 & -0.040 & 0.697 & -0.255 & 0.011 \\
CCT & -0.263 & 0.01 & 0.529 & $<0.001$ & 0.638 & $<0.001$ & N/A & N/A & 0.865 & $<0.001$ \\
CV & -0.349 & 0.001 & 0.635 & $<0.001$ & 0.579 & $<0.001$ & 0.865 & $<0.001$ & N/A & N/A \\
ACD & -0.454 & $<0.001$ & 0.180 & 0.076 & 0.123 & 0.227 & -0.103 & 0.311 & 0.009 & 0.927 \\
ACV & -0.565 & $<0.001$ & 0.158 & 0.121 & 0.087 & 0.393 & 0.024 & 0.813 & 0.073 & 0.475 \\
IOP & -0.14 & 0.915 & 0.004 & 0.966 & 0.506 & $<0.001$ & 0.295 & 0.003 & 0.182 & 0.072 \\
Spheric value & 0.262 & 0.008 & -0.148 & 0.147 & -0.121 & 0.237 & -0.172 & 0.091 & -0.163 & 0.110 \\
Cylindrical value & -0.278 & 0.007 & 0.119 & 0.244 & 0.193 & 0.056 & 0.256 & 0.011 & 0.250 & 0.013 \\
SE & 0.231 & 0.025 & -0.135 & 0.186 & -0.097 & 0.341 & -0.128 & 0.204 & -0.137 & 0.174 \\
\hline
\end{tabular}

CH: corneal hysteresis; CRF: corneal resistance factor; IOPgc: intraocular pressure (Goldman correlated); IOPcc: intraocular pressure (cornea compensated); CCT: central corneal thickness; CV: corneal volume; ACD: anterior chamber depth; ACV: anterior chamber volume; SE: spherical equivalent; IOP: intraocular pressure.

TABLE 3: The corneal curvature and keratometric values of both anterior and posterior surfaces.

\begin{tabular}{lcc}
\hline & Mean & Standard deviation \\
\hline Horizontal radius of CC (mm) & 7.86 & .27 \\
Vertical radius of CC (mm) & 7.73 & .27 \\
Average radius of CC (mm) & 7.79 & .26 \\
Anterior flat $K$ value (D) & 42.92 & 1.44 \\
Anterior steep K value (D) & 43.84 & 1.53 \\
Average anterior $K$ value (D) & 43.39 & 1.43 \\
Posterior flat $K$ value (D) & -5.96 & 1.26 \\
Posterior steep $K$ value (D) & -6.41 & .30 \\
Average posterior $K$ value (D) & -6.19 & .66 \\
Average flat $K$ value (D) & 41.67 & 1.34 \\
Average steep K value (D) & 42.50 & 1.48 \\
Average $K$ value (D) & 42.09 & 1.37 \\
Corneal astigmatism (D) & 0.86 & 0.66 \\
\hline
\end{tabular}

$K$ : keratometric; CC: corneal curvature.

are some controversial reports, most of the studies found a negative correlation between biomechanical properties and age $[3,10,13-16]$.

In the present study, it is found that $\mathrm{CH}$ and CRF are negatively correlated with age in Caucasian population. Kotecha et al. also reported that $\mathrm{CH}$ and $\mathrm{CRF}$ are negatively correlated with age in a group of patients which included patients of different ethnic origin [10]. In a recent study, Jóhannesson et al. compared 50 Swedish young subjects with 43 elderly. They reported that $\mathrm{CH}$ was significantly lower in elderly group, but there was no difference in CRF between the groups [15]. Disaccordingly, in a study from Japan, no correlation was found between age and $\mathrm{CH}$ within 86 eyes of 43 healthy subjects with an age range of 19-64 years [3]. Ortiz et al. reported significant difference in $\mathrm{CH}$ between the youngest age group ( 9 to 14 years) and oldest age group (60 to 80 years) only [16].
Central corneal thickness is one of the important geometric corneal parameters that influence IOP measurement [17]. The biomechanical properties of cornea as $\mathrm{CH}$ and $\mathrm{CRF}$ are strongly correlated with CCT $[4-6,18]$. The results of this study were in accordance with the literature. Besides CCT, the relationship of corneal curvature with corneal biomechanics was also studied. In children CC was found to be negatively correlated with $\mathrm{CH}$ and $\mathrm{CRF}[12,13,19]$. In adults, the findings of the studies that investigated CC relation with corneal biomechanics are controversial. Franco and Lira found no relationship between $\mathrm{CH}, \mathrm{CRF}$, and $\mathrm{CC}$ in adults (20-63 years of age range) in 63 eyes [8]. Kamiya et al. reported similar result; they did not find any correlation between mean $K$ value and $\mathrm{CH}$ and $\mathrm{CRF}$ in 86 eyes of 43 patients with a mean age of 39 years [3]. Wong and Lam found no correlation between mean $\mathrm{K}$ and $\mathrm{CH}$ and CRF in Chinese adults [9]. However, 2 different studies from Far East reported 
TABLE 4: The relationship of corneal biomechanical properties, age, CCT, and CV with keratometric values of anterior and posterior corneal surface.

\begin{tabular}{|c|c|c|c|c|c|c|}
\hline & & Age & $\mathrm{CH}$ & $\mathrm{CRF}$ & CCT & $\mathrm{CV}$ \\
\hline \multirow{2}{*}{ Horizontal radius of CC } & $r$ & -0.247 & -0.181 & -0.101 & 0.096 & -0.189 \\
\hline & $p$ & 0.012 & 0.085 & 0.362 & 0.351 & 0.065 \\
\hline \multirow{2}{*}{ Vertical radius of CC } & $r$ & -0.165 & -0.217 & -0.072 & 0.052 & -0.196 \\
\hline & $p$ & 0.123 & 0.033 & 0.454 & 0.589 & 0.057 \\
\hline \multirow{2}{*}{ Average radius of CC } & $r$ & -0.211 & -0.201 & -0.093 & 0.077 & -0.199 \\
\hline & $p$ & 0.034 & 0.043 & 0.387 & 0.445 & 0.047 \\
\hline \multirow{2}{*}{ Anterior flat $K$ value } & $r$ & 0.213 & 0.206 & 0.108 & -0.069 & 0.222 \\
\hline & $p$ & 0.032 & 0.046 & 0.311 & 0.532 & 0.027 \\
\hline \multirow{2}{*}{ Anterior steep $K$ value } & $r$ & 0.198 & 0.197 & 0.074 & -0.076 & 0.175 \\
\hline & $p$ & 0.055 & 0.059 & 0.503 & 0.455 & 0.095 \\
\hline \multirow{2}{*}{ Average anterior $K$ value } & $r$ & 0.209 & 0.206 & 0.095 & -0.073 & 0.204 \\
\hline & $p$ & 0.032 & 0.044 & 0.375 & 0.472 & 0.043 \\
\hline \multirow{2}{*}{ Posterior flat $K$ value } & $r$ & -0.125 & 0.182 & 0.128 & 0.157 & 0.078 \\
\hline & $p$ & 0.240 & 0.091 & 0.208 & 0.119 & 0.443 \\
\hline \multirow{2}{*}{ Posterior steep $K$ value } & $r$ & -0.103 & -0.362 & -0.254 & -0.143 & -0.478 \\
\hline & $p$ & 0.287 & $<0.001$ & 0.013 & 0.152 & $<0.001$ \\
\hline \multirow{2}{*}{ Average posterior $K$ value } & $r$ & -0.143 & 0.085 & 0.065 & 0.123 & -0.034 \\
\hline & $p$ & 0.175 & 0.419 & 0.528 & 0.254 & 0.737 \\
\hline \multirow{2}{*}{ Average flat $K$ value } & $r$ & 0.160 & 0.184 & 0.069 & -0.095 & 0.183 \\
\hline & $p$ & 0.103 & 0.085 & 0.497 & 0.342 & 0.069 \\
\hline \multirow{2}{*}{ Average steep $K$ value } & $r$ & 0.243 & 0.156 & 0.047 & -0.151 & 0.080 \\
\hline & $p$ & 0.019 & 0.125 & 0.642 & 0.138 & 0.489 \\
\hline \multirow{2}{*}{ Average $K$ value } & $r$ & 0.212 & 0.175 & 0.060 & -0.127 & 0.129 \\
\hline & $p$ & 0.034 & 0.088 & 0.560 & 0.207 & 0.201 \\
\hline \multirow{2}{*}{ Corneal astigmatism } & $r$ & 0.192 & -0.048 & -0.092 & -0.165 & -0.203 \\
\hline & $p$ & 0.061 & 0.702 & 0.419 & 0.108 & 0.041 \\
\hline
\end{tabular}

K: keratometric; CC: corneal curvature.

contradictory results. Narayanaswamy et al. found that flatter and thinner corneas were related to lower $\mathrm{CH}$ and $\mathrm{CRF}$ in 1136 Chinese patients (mean age 55 years) [2]. Hwang et al. reported results of 958 eyes of 958 patients with a mean age of 27 years. They concluded that $\mathrm{CH}$ and $\mathrm{CRF}$ were negatively correlated with mean radius of curvature [20]. Kotecha et al. found similar results in patients with different races with a mean age 50 years [10]. In other studies only $\mathrm{CH}$ was found to be associated with mean $K[11,21]$. Diversity of the results in these studies may be related to the racial variety of the patients and age groups. Another reason for these results may be due to the different devices used for measurement for corneal parameters.

Apart from the other studies, this study investigated both steep and flat $K$ values of anterior and posterior surface of the cornea. Although no correlation was found between mean $K$ value and $\mathrm{CH}$ and $\mathrm{CRF}$, it is noted that there are correlations with posterior steep $K$ value and vertical radius of CC. CRF was also correlated with mean posterior $K$ value and posterior steep $K$ value. According to a multivariate regression model in the present study, posterior $K$ values were found to be associated with $\mathrm{CH}$ and $\mathrm{CRF}$. This finding may suggest that posterior surface of the cornea may affect corneal biomechanical properties more compared to anterior surface in normal eyes. In a recent study, it has been shown that $\mathrm{CH}$ and CRF were negatively associated with $K$ max value in keratoconic eyes, but this correlation did not exist for normal eyes [22].

$\mathrm{CV}$ and its relation with corneal biomechanical properties have also been investigated in normal and keratoconic eyes in several studies $[23,24]$. Hwang et al. found that corneal volume was positively correlated with $\mathrm{CH}$, but not CRF [20]. Wong and Lam reported positive correlation both with $\mathrm{CH}$ and CRF [9]. In a recent study both $\mathrm{CH}$ and $\mathrm{CRF}$ were found to be correlated positively with $\mathrm{CC}$ and $\mathrm{CV}$ for the normal, keratoconic, and crosslinked eyes [22]. In this study, there are also positive correlations noted between $\mathrm{CH}, \mathrm{CRF}$, and $\mathrm{CV}$.

In conclusion, $\mathrm{CH}$ and $\mathrm{CRF}$ are positively correlated with CCT and CV and negatively correlated with age in a healthy Caucasian population. It is also shown with this study that $\mathrm{CH}$ and $\mathrm{CRF}$ are negatively correlated with posterior steep and average posterior $K$ values. Anterior and posterior $K$ values account for $16.6 \%$ and $9.5 \%$ of variation in $\mathrm{CH}$ and CRF, respectively. 


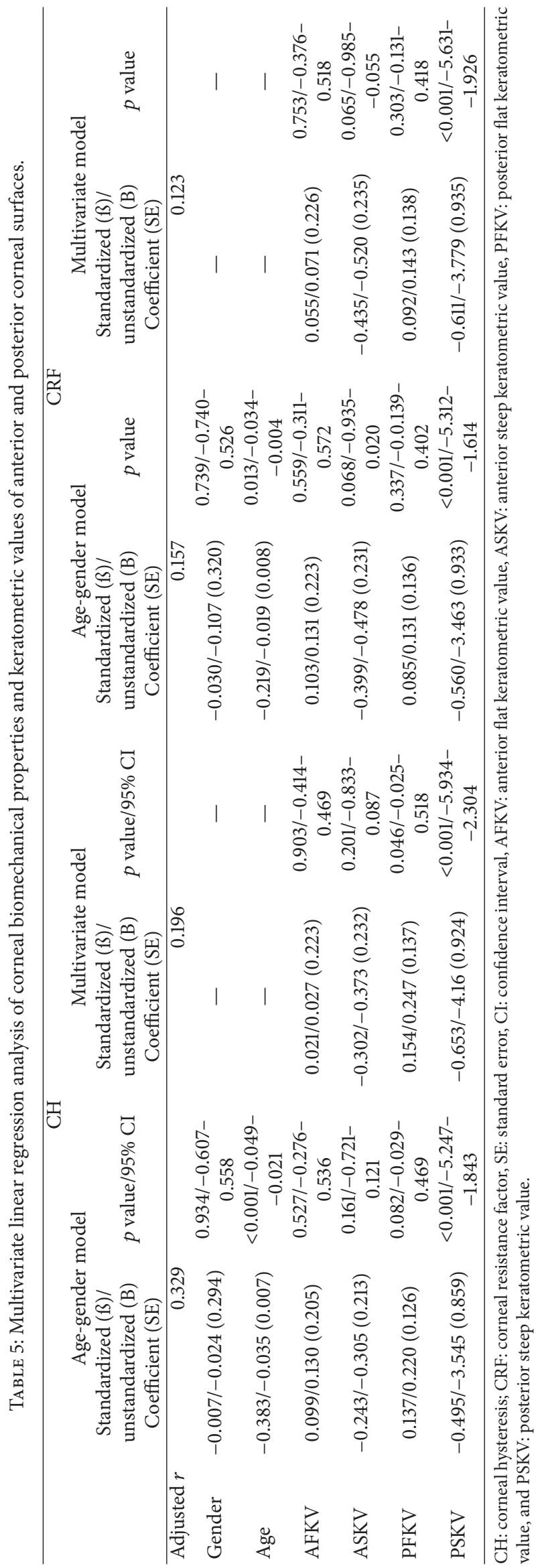




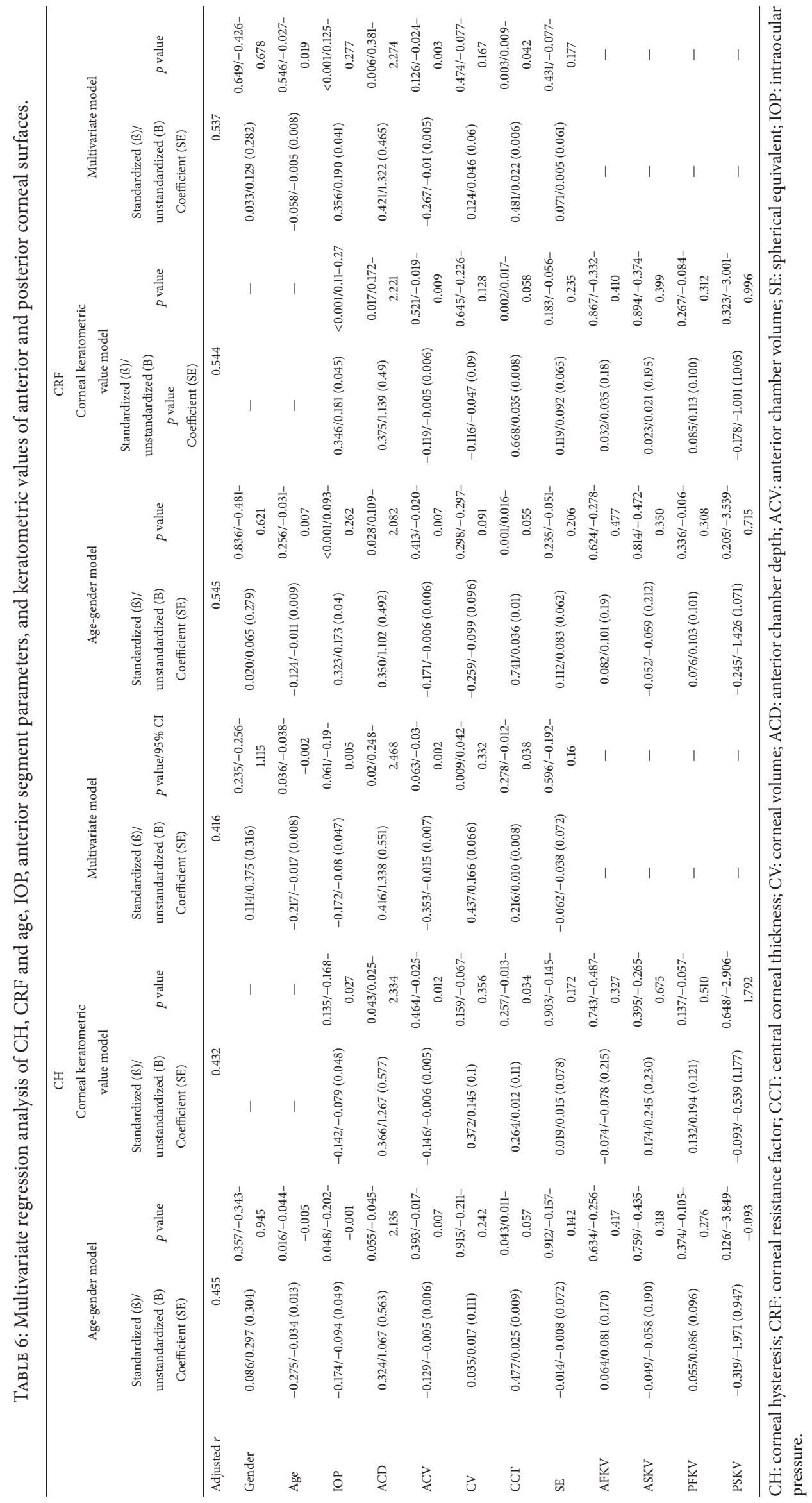




\section{Competing Interests}

None of the authors has conflict of interests with regard to submission.

\section{References}

[1] N. Terai, F. Raiskup, M. Haustein, L. E. Pillunat, and E. Spoerl, "Identification of biomechanical properties of the cornea: the ocular response analyzer," Current Eye Research, vol. 37, no. 7, pp. 553-562, 2012.

[2] A. Narayanaswamy, R. S. Chung, R.-Y. Wu et al., "Determinants of corneal biomechanical properties in an adult Chinese population," Ophthalmology, vol. 118, no. 7, pp. 1253-1259, 2011.

[3] K. Kamiya, M. Hagishima, F. Fujimura, and K. Shimizu, "Factors affecting corneal hysteresis in normal eyes," Graefe's Archive for Clinical and Experimental Ophthalmology, vol. 246, no. 10, pp. 1491-1494, 2008.

[4] D. Touboul, C. Roberts, J. Kérautret et al., "Correlations between corneal hysteresis, intraocular pressure, and corneal central pachymetry," Journal of Cataract \& Refractive Surgery, vol. 34, no. 4, pp. 616-622, 2008.

[5] M. Detry-Morel, J. Jamart, and S. Pourjavan, "Evaluation of corneal biomechanical properties with the Reichert Ocular Response Analyzer," European Journal of Ophthalmology, vol. 21, no. 2, pp. 138-148, 2011.

[6] G. Mangouritsas, G. Morphis, S. Mourtzoukos, and E. Feretis, "Association between corneal hysteresis and central corneal thickness in glaucomatous and non-glaucomatous eyes," Acta Ophthalmologica, vol. 87, no. 8, pp. 901-905, 2009.

[7] M. A. del Buey, L. Lavilla, F. J. Ascaso, E. Lanchares, V. Huerva, and J. A. Cristóbal, "Assessment of corneal biomechanical properties and intraocular pressure in myopic Spanish healthy population," Journal of Ophthalmology, vol. 2014, Article ID 905129, 6 pages, 2014.

[8] S. Franco and M. Lira, "Biomechanical properties of the cornea measured by the Ocular Response Analyzer and their association with intraocular pressure and the central corneal curvature," Clinical and Experimental Optometry, vol. 92, no. 6, pp. 469-475, 2009.

[9] Y. Z. Wong and A. K. Lam, "Influence of corneal astigmatism, corneal curvature and meridional differences on corneal hysteresis and corneal resistance factor," Clinical and Experimental Optometry, vol. 94, no. 5, pp. 418-424, 2011.

[10] A. Kotecha, R. A. Russell, A. Sinapis, S. Pourjavan, D. Sinapis, and D. F. Garway-Heath, "Biomechanical parameters of the cornea measured with the Ocular Response Analyzer in normal eyes," BMC Ophthalmology, vol. 14, article 11, 2014.

[11] N. Rosa, M. Lanza, M. De Bernardo, G. Signoriello, and P. Chiodini, "Relationship between corneal hysteresis and corneal resistance factor with other ocular parameters," Seminars in Ophthalmology, vol. 30, no. 5-6, pp. 335-339, 2015.

[12] L. Lim, G. Gazzard, Y. H. Chan et al., "Cornea biomechanical characteristics and their correlates with refractive error in Singaporean children," Investigative Ophthalmology and Visual Science, vol. 49, no. 9, pp. 3852-3857, 2008.

[13] I. Bueno-Gimeno, E. España-Gregori, A. Gene-Sampedro, A. Lanzagorta-Aresti, and D. P. Piñero-Llorens, "Relationship among corneal biomechanics, refractive error, and axial length," Optometry and Vision Science, vol. 91, no. 5, pp. 507-513, 2014.
[14] A. Kotecha, A. Elsheikh, C. R. Roberts, H. Zhu, and D. F Garway-Heath, "Corneal thickness- and age-related biomechanical properties of the cornea measured with the ocular response analyzer," Investigative Ophthalmology and Visual Science, vol. 47, no. 12, pp. 5337-5347, 2006.

[15] G. Jóhannesson, P. Hallberg, K. Ambarki, A. Eklund, and C. Lindén, "Age-dependency of ocular parameters: a cross sectional study of young and elderly healthy subjects," Graefe's Archive for Clinical and Experimental Ophthalmology, vol. 253, no. 11, pp. 1979-1983, 2015.

[16] D. Ortiz, D. Piñero, M. H. Shabayek, F. Arnalich-Montiel, and J. L. Alió, "Corneal biomechanical properties in normal, postlaser in situ keratomileusis, and keratoconic eyes," Journal of Cataract and Refractive Surgery, vol. 33, no. 8, pp. 1371-1375, 2007.

[17] J. D. Brandt, J. A. Beiser, M. A. Kass, and M. O. Gordon, “Central corneal thickness in the Ocular Hypertension Treatment Study (OHTS)," Ophthalmology, vol. 108, no. 10, pp. 1779-1788, 2001.

[18] B. Akova-Budak and S. A. Kıvanç, "Does corneal hysteresis correlate with endothelial cell density?" Medical Science Monitor, vol. 21, pp. 1460-1463, 2015.

[19] P.-Y. Chang, S.-W. Chang, and J.-Y. Wang, "Assessment of corneal biomechanical properties and intraocular pressure with the Ocular Response Analyzer in childhood myopia," British Journal of Ophthalmology, vol. 94, no. 7, pp. 877-881, 2010.

[20] H. S. Hwang, S. K. Park, and M. S. Kim, "The biomechanical properties of the cornea and anterior segment parameters," BMC Ophthalmology, vol. 13, article 49, 2013.

[21] A. T. Broman, N. G. Congdon, K. Bandeen-Roche, and H. A. Quigley, "Influence of corneal structure, corneal responsiveness, and other ocular parameters on tonometric measurement of intraocular pressure," Journal of Glaucoma, vol. 16, no. 7, pp. 581-588, 2007.

[22] D. Viswanathan, N. L. Kumar, J. J. Males, and S. L. Graham, "Relationship of structural characteristics to biomechanical profile in normal, keratoconic, and crosslinked eyes," Cornea, vol. 34, no. 7, pp. 791-796, 2015.

[23] V. Kozobolis, H. Sideroudi, A. Giarmoukakis, M. Gkika, and G. Labiris, "Corneal biomechanical properties and anterior segment parameters in forme fruste keratoconus," European Journal of Ophthalmology, vol. 22, no. 6, pp. 920-930, 2012.

[24] B. M. Fontes, R. Ambrósio Jr., D. Jardim, G. C. Velarde, and W. Nosé, "Corneal biomechanical metrics and anterior segment parameters in mild keratoconus," Ophthalmology, vol. 117, no. 4, pp. 673-679, 2010. 


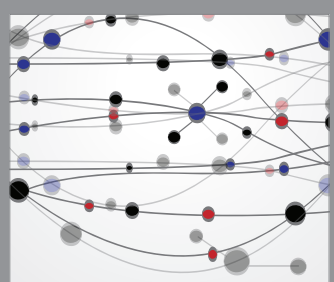

The Scientific World Journal
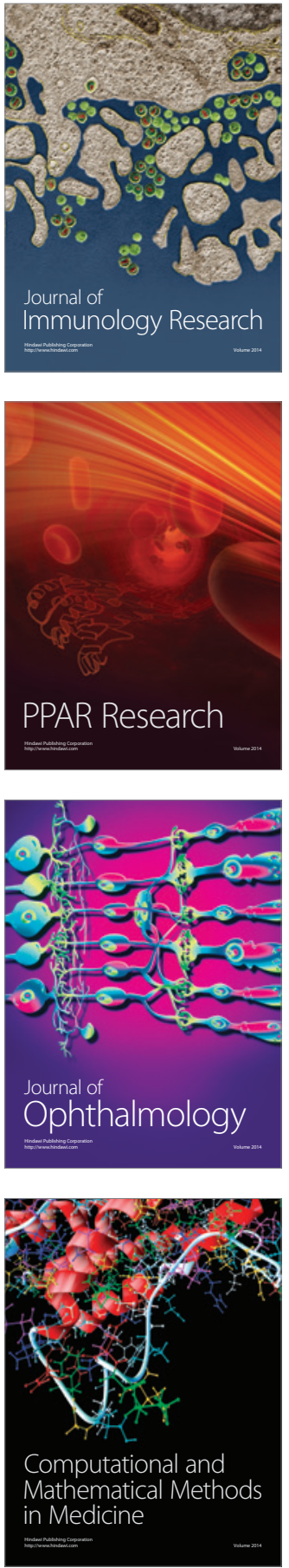

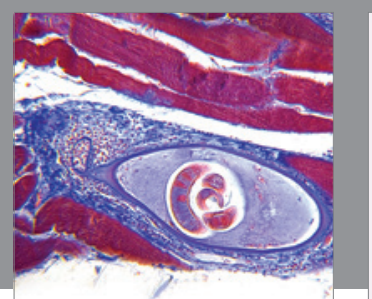

Gastroenterology Research and Practice

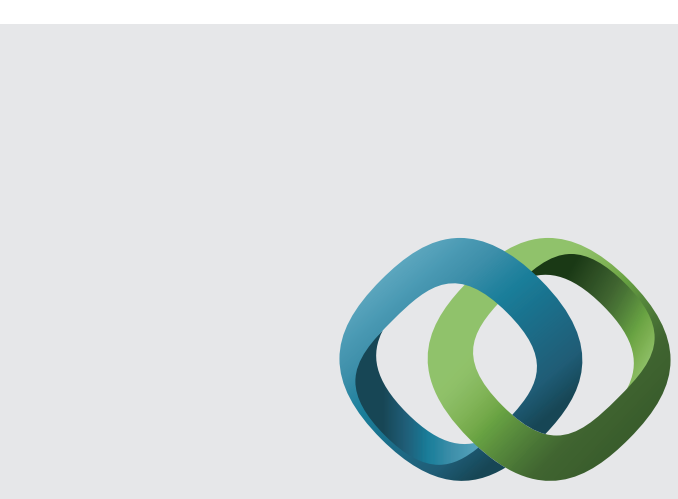

\section{Hindawi}

Submit your manuscripts at

http://www.hindawi.com
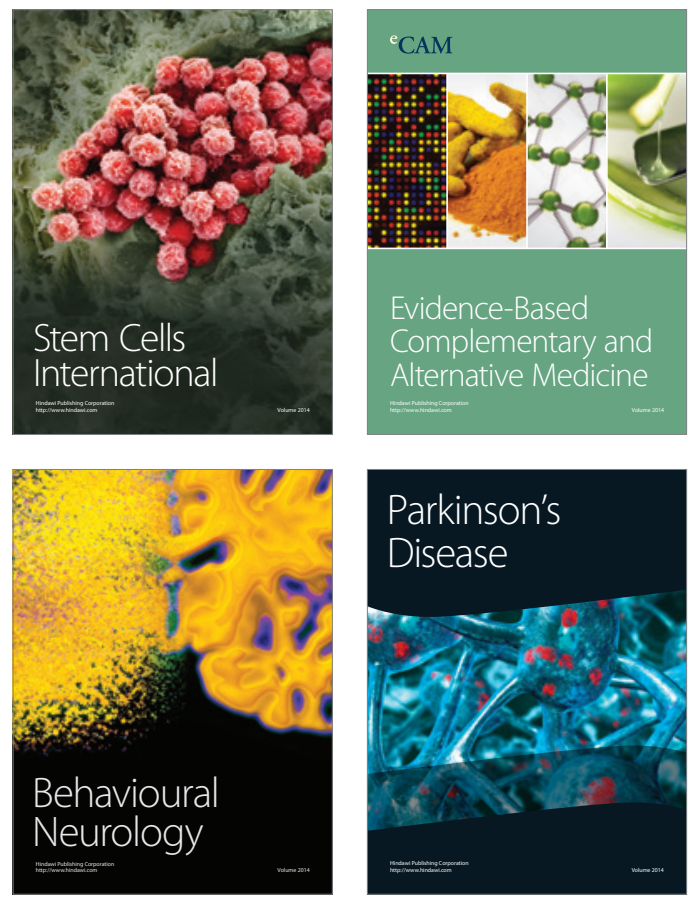
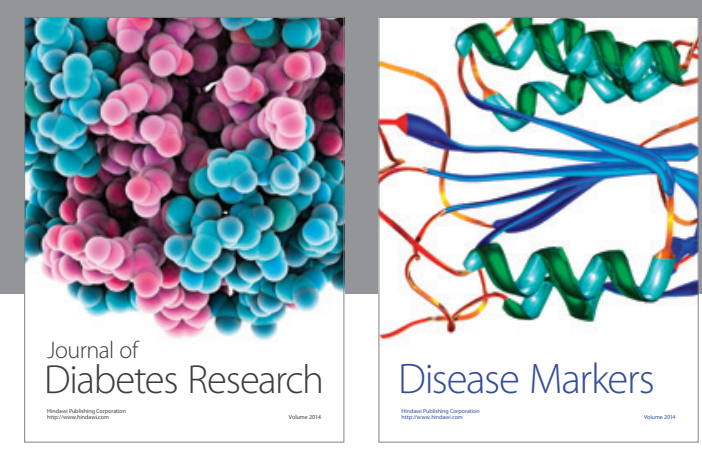

Disease Markers
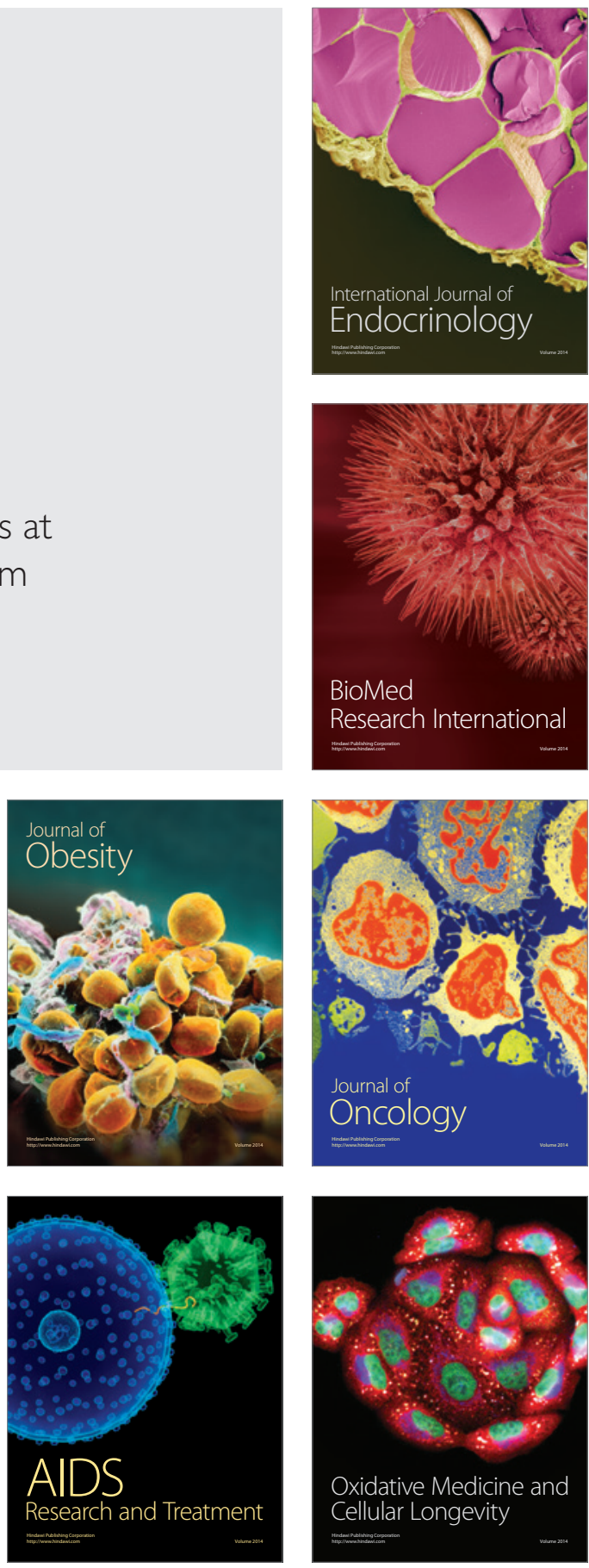